\title{
SCENTED CANDLES AS A SOURCE OF NANOPARTICLES IN THE WORKSPACE
}

\author{
${ }^{1}$ Petra ROUPCOVÁ, ${ }^{2}$ Hana KUBÁTOVÁ, ${ }^{1,3}$ Karel KLOUDA, ${ }^{1}$ Ondřej CHROMEČKA
}

\author{
${ }^{1}$ VSB - Technical University of Ostrava, Ostrava, Czech Republic, EU, \\ petra.roupcova@vsb.cz; karel.klouda@vsb.cz; ondrej.chromecka@vsb.cz \\ ${ }^{2}$ State Office for Nuclear Safety, Prague, Czech Republic, EU, hana.kubatova@sujb.cz \\ ${ }^{3}$ Research Institute for Occupational Health and Safety, Prague, EU, klouda@vubp-praha.cz
}

https://doi.org/10.37904/nanocon.2020.3759

\begin{abstract}
Scented candles are used in many professions. Both gaseous and solid emissions are generated in the workplace by their combustion. In our pilot experiment, we focused on determining the concentration of nanoparticles in the environment, their mean diameter, and lung deposited surface area (LSDA). The article presents the results obtained during the measurement in areas simulating a dry environment (shop, restaurant) and a humid environment (spa, rehabilitation). The concentrations of nanoparticles in the area of the simulation workplace (area $5.3 \mathrm{~m}^{2}$, volume $12.7 \mathrm{~m}^{3}$ ) during the burning of a single candle were measured to be ca. 1600 thousand particles in $\mathrm{cm}^{3}$ in the atmosphere. Such values are comparable to a foundry, engineering plant or glassworks. Measured concentrations can pose a risk primarily to both employees and clients. While in industrial plants the risks arising from the presence of dust and nanoparticles are taken into account and workers are provided protective equipment, in shops, spas or beauty salons, where scented candles are burned, they are often neglected.
\end{abstract}

Keywords: Scented candles, emissions, nanoparticles, measurements, precaution

\section{INTRODUCTION}

Scented candles are a very popular item. They are used not only in households but also in stores, beauty salons, relaxation centers, cafes and restaurants, where their scent contributes to creating a pleasant atmosphere and the sense of well-being and relaxation which accompany the services offered. Burning scented candles releases a large amount of emissions into the environment, both gaseous and solid (particles). The composition of the emissions depends on the composition of the individual components of candles. The main components of candles are wax and wick; others components include aromatic substances (scents) and dyes or pigments. [1,2] Wax is the fuel that burns in candles. The origin of the wax used, the method of production and its chemical composition influence the composition of emissions. The most common types of wax from which candles are made are paraffin and stearin, or mixtures thereof. Beeswax and vegetable waxes are used less mainly because of their cost. Paraffin is a mixture of paraffinic hydrocarbons obtained by processing petroleum and may contain residues of aliphatic and aromatic hydrocarbons, and a variety of other substances. Stearin (tristearin) is a triglyceride derived from three units of stearic acid, and it is produced by processing animal fats and vegetable oils [3]. In addition to the fuel (wax), the composition of emissions is also affected by volatile substances which give scented candles their aroma. Synthetic scents in particular cause problems. Laboratory analyses carried out in studies on the composition of emissions from the burning of scented candles have shown that the emissions include volatile organic compounds (VOCs), such as toluene, benzene or ethylbenzene, polycyclic aromatic hydrocarbons (PAH) and aldehydes. These are often toxic substances that can have a negative impact on the health of exposed persons $[1,2]$, and thus on the quality of the working environment where candles are used. Particulate matter (PM) is also a significant part of the emissions that can affect health. When burning candles, a wide range of respirable particles is formed, from coarse particles $\left(\mathrm{PM}_{10}\right)$ and fine particles $\left(\mathrm{PM}_{2.5}\right)$ to ultrafine particles (UFP PM0.1), resp. nanoparticles [3-5,7]. 
Dr. Anne Steinemann, a leading environmental scientist, has presented her studies on aromatic products (perfume fragrances) in a number of publications. Even a small amount of a fragrance component can have demonstrably negative effects on human health [6]. Candles made from natural oils and waxes - beeswax, soy, rapeseed, olive, sunflower or coconut oil - can be friendlier for the environment (work and living). However, even in these more ecological variants, the presence of synthetic additives can negatively affect the health safety of the candles.

\section{EXPERIMENTAL PART}

\subsection{Materials and methods}

The Yankee Candle Company and Woodwick scented candles, namely WoodWick (Rose), WoodWick (Warm Wool, Petite candle) and Yankee candle (A Calm \& Quiet Place), were selected for measurement. Not only the authors of the article selected the candles for measuring the nanoparticle concentrations, but the choice of candles was consulted with the sellers in the stone candle shop to make them generally popular scents that are often sold. WoodWick candles are made of a mixture of soy wax and paraffin which according to the manufacturer ensures a cleaner burning without smoke. Their wick is wooden [8]. The Yankee candle is made of paraffin wax of first-class quality, and the wick is $100 \%$ cotton, lead-free [9].

The workplaces where employees can come into contact with emissions from burning scented candles were simulated in our experiments by a bathroom - a room with two windows, an area of $5.28 \mathrm{~m}^{2}$ and a volume of $12.672 \mathrm{~m}^{3}$. Open windows provided natural ventilation during all measurements (similar to small shops and massage rooms). The experiments were performed in dry and humid environments. The arrangement of the experiments and positions for the placement of candles and measuring techniques are shown in Figure 1.

For the measurement, we used a portable Testo DiSCmini (Testo Ltd.) designed to measure the number of nanoparticles and the average size of nanoparticles with a time resolution of 1 second. At the same time, the device determined the surface area of nanoparticles present in the measured space (LDSA). As part of the measurement, we compared the change in the number of particles (measured for particles in the range of 10 to $700 \mathrm{~nm}$ ) and the size of nanoparticles before and after ignition of the selected scented candles in specified measuring positions, and the total surface area of settled particles in the alveoli. The obtained results are presented graphically as change in the number of particles in a volume unit of the atmosphere over time, change in the size of particles over time, and change in the area of particles over time.

The measurement always took place for a total of $11 \mathrm{~min}$, with a distance of $20-30 \mathrm{~cm}$ from the candle and consisted of three parts: phase No. 1 (measurement length 2 min before lighting the candle), phase No. 2 (measurement length 6 min when a scented candle was burning) and phase No. 3 (measurement duration 3 min after extinguishing the candle).

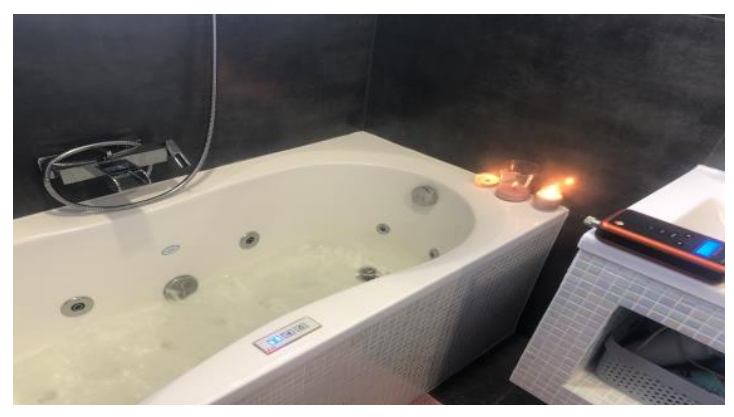

Figure 1 Arrangement of the experimental room, position of the candles and measuring technique (experiment with three burning candles in a humid environment).

\subsection{Arrangement of the experiment for a dry (normal) working environment}

For the purpose of simulating exposure in a dry working environment, a background measurement was first performed in the space reserved for the experiment without burning candles. No person was present in the room during the measurement. Measurement conditions: pressure $985.5 \mathrm{hPa}$, temperature $26.1^{\circ} \mathrm{C}$, and relative humidity $53.2 \%$. Subsequently, measurements were performed with individual scented candles placed on the edge of the bath. The final $4^{\text {th }}$ measurement in a dry environment was to verify the synergistic effect. Three candles were lit during the measurement to simulate the realistic effect they would have in wellness 
facilities. The measuring device was placed at a distance of about $20-30 \mathrm{~cm}$ from the burning medium (see Figure 1). During the measurement, the operator of the device was present in the room.

\subsection{Arrangement of the experiment for a humid working environment}

To simulate exposure in a humid working environment, a bath was filled with water in an experimental room and background measurements were performed. A person was present in the room during the measurement. Measurement conditions: pressure $985.1 \mathrm{hPa}$, temperature $25.2-26.3^{\circ} \mathrm{C}$, and relative humidity in the range of $55.3-80 \%$. A total of 2 measurements were performed with a Yankee candle (A Calm \& Quiet Place), and with 3 candles lit simultaneously placed on the edge of the tub.

\section{RESULTS AND DISCUSSION}

The measurement results of individual candles are presented graphically as the change in the number of particles (measured for particles with a size of 10 to $700 \mathrm{~nm}$ ) in a volume unit of atmosphere over time, the change in particle size over time, and the change in particle area over time. The graphs (see Figures 2-6) are divided into 3 phases: phase No. 1 (measurement length 2 min without candle burning), phase No. 2 (measurement length 6 min with scented candle burning), and phase No. 3 (measurement length 3 min after extinguishing the candle). All measurements of nanoparticle concentrations (Figures 2, 4) showed an increase in the concentration of nanoparticles after lighting the candle in phase 2. The largest increase in concentration was recorded during the measurement of Woodwick Rose candle with up to 350,000 particles $/ \mathrm{cm}^{3}$. The increase in the concentration of nanoparticles during the measurement was reflected in a decrease in their mean size (Figures 3, 5). A difference between dry and humid environments can be observed. In the humid environment there was almost a $3 x$ increase in nanoparticle concentrations (Figures 2, 4). Burning the Yankee candle (A Calm \& Quiet Place) increased the concentration from 14,000 particles $/ \mathrm{cm}^{3}$ (dry medium) to 58,000 particles $/ \mathrm{cm}^{3}$ (humid medium), while three candles burning simultaneously increased the average concentration from 170,000 particles $/ \mathrm{cm}^{3}$ (dry medium) to 550,000 particles $/ \mathrm{cm}^{3}$ (humid medium). The LungDeposited Surface Area (LDSA) measured during the burning of 3 candles in a dry environment was 117 $\mu \mathrm{m}^{2} / \mathrm{cm}^{3}$, and in a humid environment $312 \mu \mathrm{m}^{2} / \mathrm{cm}^{3}$ (Figure 6).

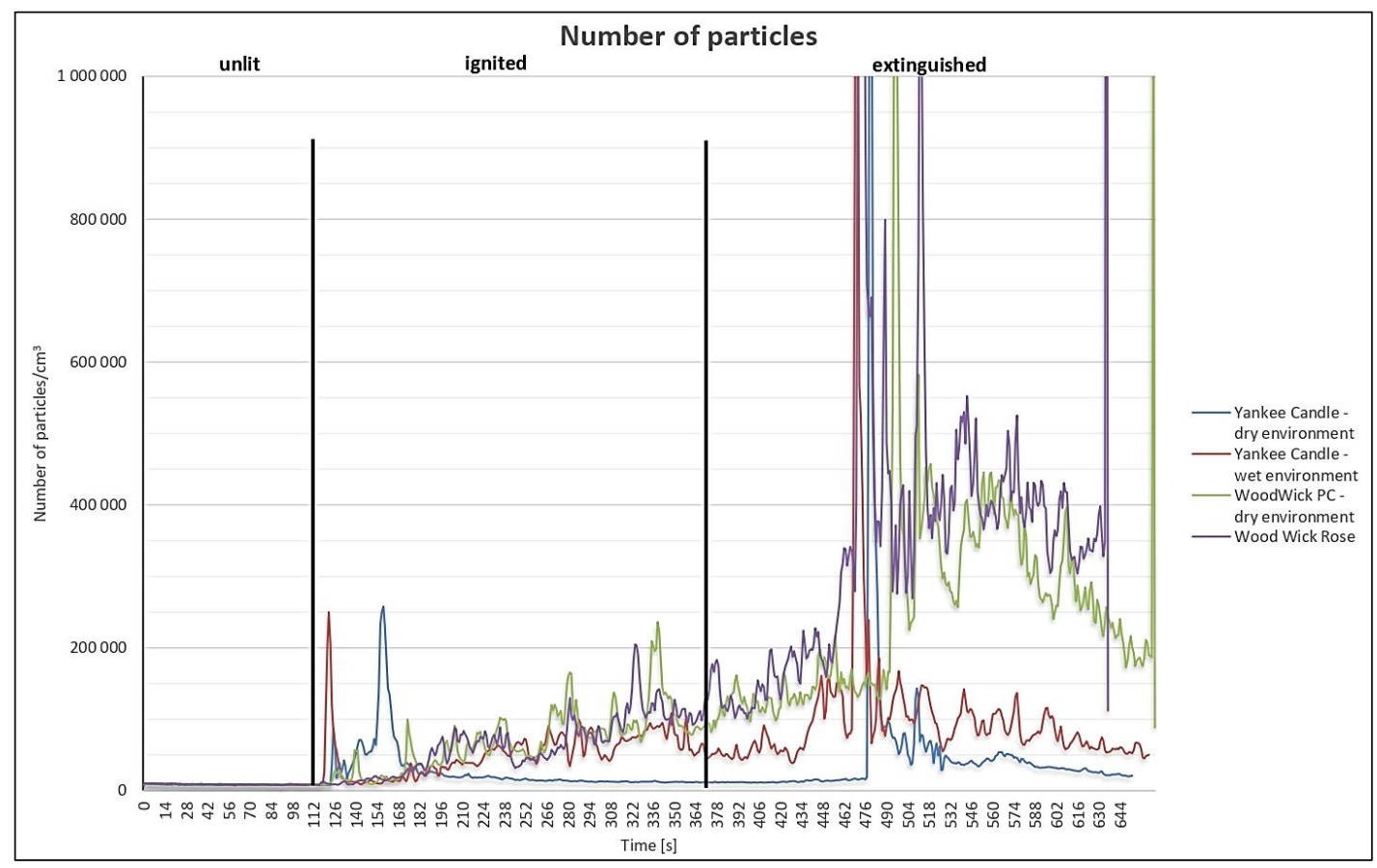

Figure 2 Nanoparticle concentration in dry and humid working environments with a burning candle during experiments (four experiments) 


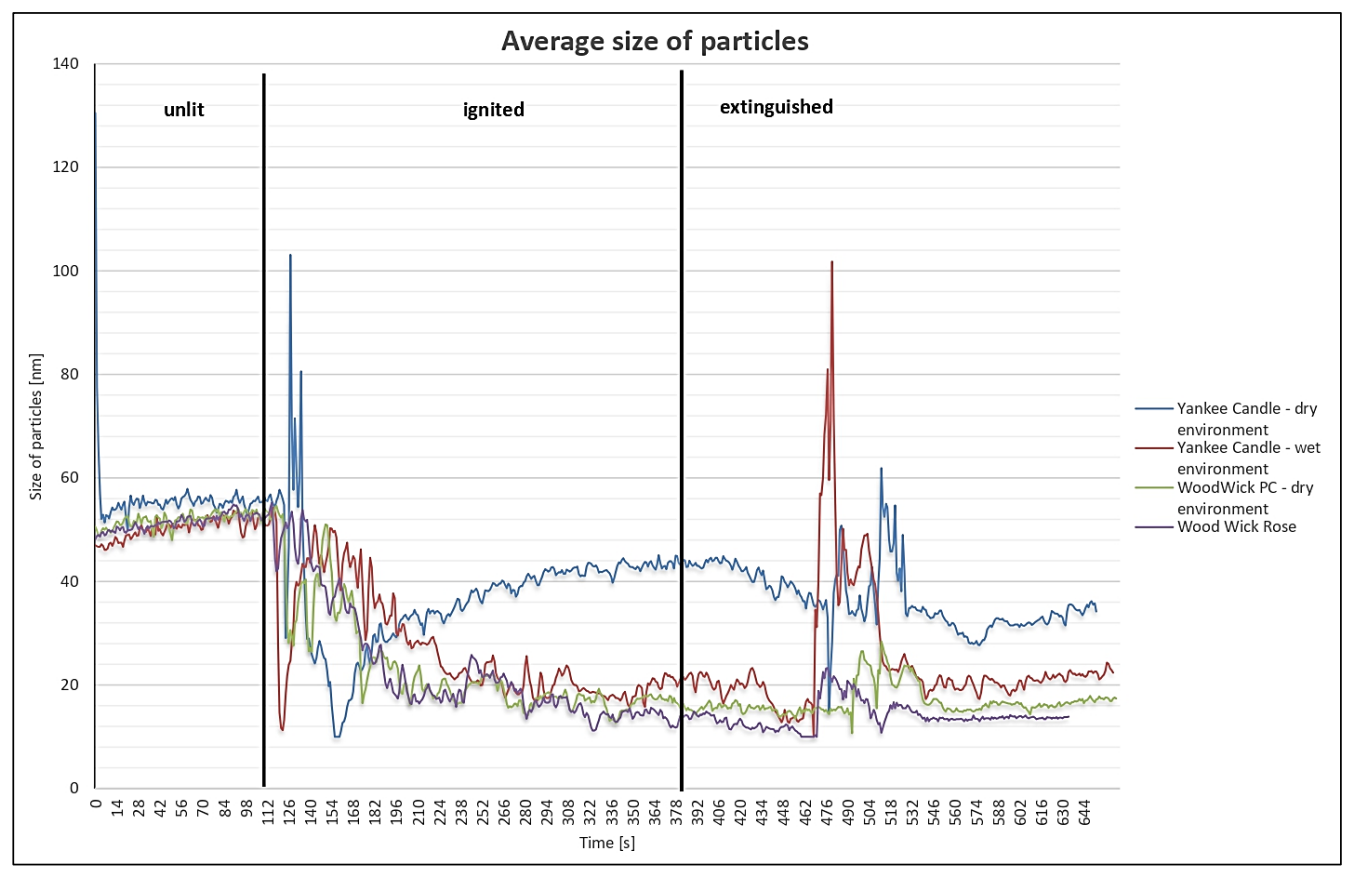

Figure 3 The average diameter of nanoparticles in dry and humid working environments with a burning candle during the experiment - Yankee candle (four experiments)

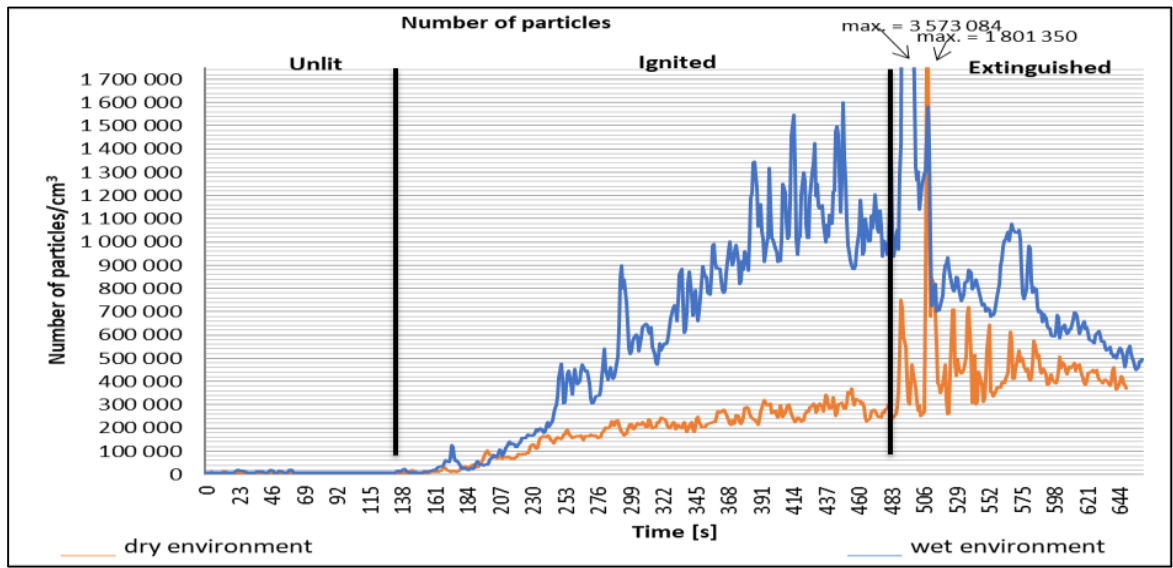

Figure 4 Nanoparticle concentration in dry and humid environments where 3 scented candles burn simultaneously (Yankee candle (A calm and quiet place), WoodWick Rose, Woodwick Petite candle)

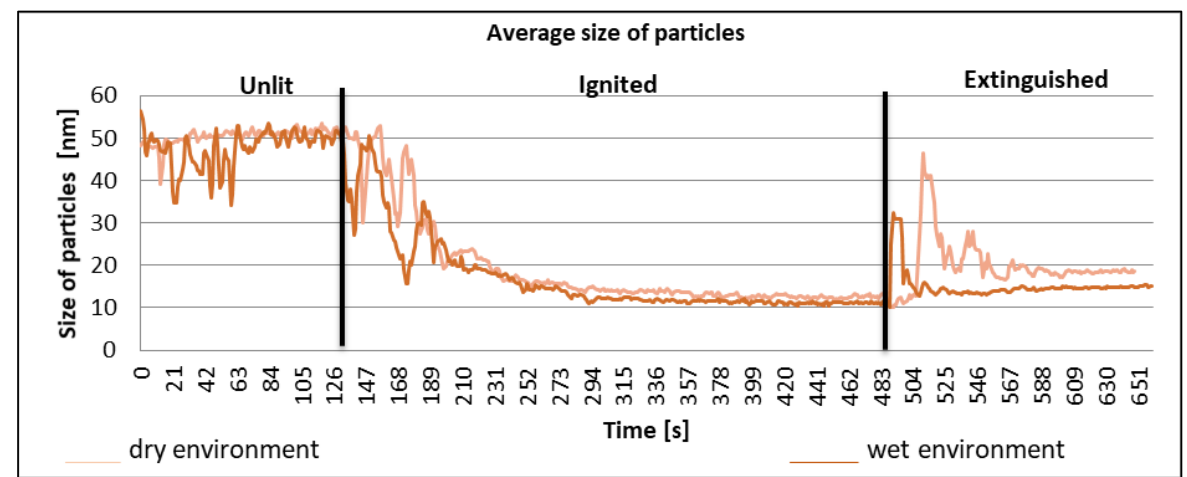

Figure 5 The average size of nanoparticles in dry and humid environments where 3 scented candles burn simultaneously (Yankee candle (A calm and quiet place), WoodWick Rose, Woodwick Petite candle) 


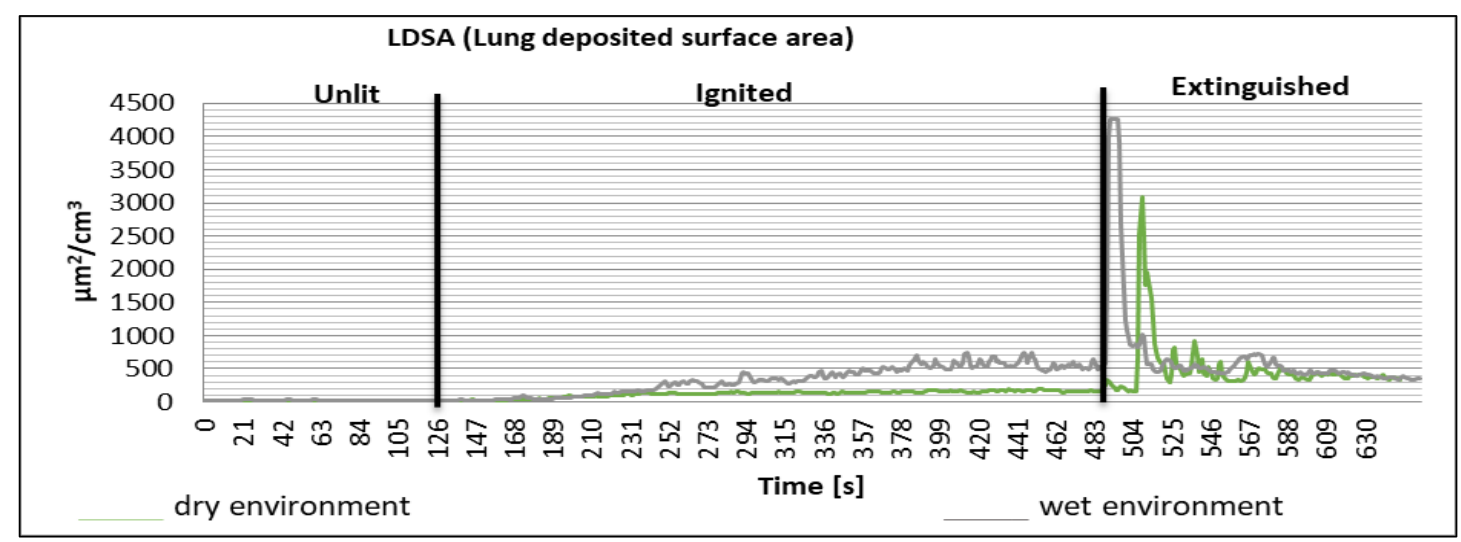

Figure 6 LDSA in dry and humid environments where 3 scented candles burn simultaneously (Yankee candle (A calm and quiet place), WoodWick Rose, Woodwick Petite candle).

\section{CONCLUSION}

The measurement of a set of three types of scented candles took place first in a bathroom with an empty bathtub and then with a proband and a bathtub full of water. In all measurements of nanoparticle concentrations there was an increase in the concentration of nanoparticles after lighting the candle in phase 2 . The largest increase in the concentration of nanoparticles was measured when burning a WoodWick scented candle - a scented rose in a dry environment. Further, most of all measurements showed an increase in the concentration of nanoparticles formed during the burning of scented candles in a humid environment compared to a dry environment. A detailed assessment of the influence of the type of wax and wick on burning scented candles has not been investigated in more detail during the experiments. From the measured data it is evident that the concentration of measured nanoparticles was higher in two performed experiments for humid environment compared to dry environment. Combustion products were formed during the burning of scented candle. The reaction conditions were changed (humid and dry environment) subsequently different combustion products were formed. These products might have a hydrophilic or hydrophobic character that could explain the differences between measured concentrations of nanoparticles. As the concentration of nanoparticles increased, the mean diameter of the nanoparticles decreased. These results show that the measured concentrations of nanoparticles in the bathroom atmosphere when burning a scented candle have values even higher than in engineering companies, foundries and glassworks during the manual processing of glass.

\section{REFERENCES}

[1] PETRY, T., VITALE D., JOACHIM F. J., SMITH B., CRUSE L., MACHARENHAS R., SCHNEIDER S., SIGAL M. Human health risk evaluation of selected VOC, SVOC and particulate emissions from scented candles. Regulatory Toxicology and Pharmacology. 2014, vol. 69, no.1, pp. 55-70.

[2] DERUDI M., GELOSA S., SLIEPCEVICH A., CATTANEO A., ROTA R., CAVALLO D., NANO G. Emissions of air pollutants from scented candles burning in a test chambre. Atmospheric Environment. 2012, vol. 55, pp. $257-262$.

[3] SOPPA, V. J., SCHINS, R. P., HENNING, F., HELLACK, B., QUASS, U., KAMINSKI, H., KUHLBUSCH, T. A., HOFFMAN, B., WEINMAYR, G. Respiratory effects of fine and ultrafine particles from indoor sources--a randomized sham-controlled exposure study of healthy volunteers. International journal of environmental research and public health. 2014, vol. 11, no. 7, pp. 6871-6889.

[4] THE DANISH ENVIRONMENTAL PROTECTION AGENCY. Environmentally friendly candles with reduced particle emissions [online]. [Viewed 2019-11-20]. Available from: https://www2.mst.dk/Udgiv/publications/2018/11/978-87-7038-009-6.pdf.

[5] ANEC position paper. ANEC recommendations on the emissions to the indoor air. [Viewed 2020-04-20]. Available from: https://www.anec.eu/images/Publications/position-papers/Chemicals/ANEC-PT-2015-CEG016.pdf. 
[6] STEINEMANN, A., GOODMAN. N. Fragranced consumer products and effects on asthmatics: an international population-based study. Air Quality, Atmosphere \& Health. 2019, vol. 12, no. 6, pp. 643-649.

[7] CSN EN15426/2019 (650122) Candles - Specification for the determination of the smokyness of candles.

[8] WOODWICK SVICKY. [Viewed 2019-12-02]. Available from: https://woodwick.svicky.cz/woodwick-rose-vazastredni.html.

[9] VERYBERRY. [Viewed 2019-11-04]. Available from: https://veryberry.cz/vonne-a-klasicke-svicky/8798-yc-sklo-1a-calm-quiet-place-5038581033327.html. 